\title{
Anatomia Digital: um Ambiente Virtual de Apoio ao Processo Ensino-aprendizagem
}

\author{
Alexsandro R. Silva ${ }^{1}$, Matheus M. C. Valerio ${ }^{1}$, Paulo A. M. Neto ${ }^{1}$, Amadeu S \\ Campos Filho ${ }^{1,2}$ \\ ${ }^{1}$ Faculdade São Miguel, Recife - PE, 50070-070, Brasil \\ ${ }^{2}$ Núcleo de Telessaúde - Hospital das Clínicas - Universidade Federal de Pernambuco \\ (UFPE), Recife, PE - Brasil \\ \{thealeehmy, matheus.moura13\}@gmail.com, arquivopauloneto@hotmail.com, \\ amadeu.campos@nutes.ufpe.br
}

\begin{abstract}
The process of learning the discipline of human anatomy is complex, exhaustive and highly detailed. In this scenario, this study aims to describe the process of design, development and evaluation of a virtual environment to support the teaching-learning process of human anatomy. The methodology used was based on software engineering, user-centered design and usability assessment. As a project results, an environment with virtual reality technology was developed and based on gamification theories. In addition, the usability test in the virtual environment occurred in a normal and pleasant way in the view of the users, who demonstrated positivity for the future of the project.
\end{abstract}

Resumo. O processo de aprendizado da disciplina de anatomia humana é complexo, exaustivo e altamente detalhista. Nesse cenário, este estudo tem como objetivo descrever o processo de design, desenvolvimento e avaliação de um ambiente virtual de apoio ao processo ensino-aprendizagem da disciplina de anatomia humana. A metodologia utilizada foi baseada na engenharia de software, design centrado no usuário e avaliação de usabilidade. Como resultado do projeto, foi desenvolvido um ambiente com a tecnologia de realidade virtual e baseado nas teorias de gamificação. Além disso, o teste de usabilidade no ambiente virtual ocorreu de forma normal e agradável na visão dos usuários, que demonstraram positividade para o futuro do projeto.

\section{Introdução}

O processo de ensino-aprendizagem tem sofrido várias mudanças importantes ao decorrer da história. Antigamente o professor tinha a tarefa árdua e cansativa de ensinar todo conteúdo para seus alunos, pois o professor era o detentor do conhecimento e as metodologias de aprendizagem eram baseadas no comportamento do aluno diante aos conteúdos que o professor lecionasse. Muitas vezes, esses conteúdos eram complexos, numerosos e possuíam várias nuances difíceis de serem compreendidas. Além disso, os conteúdos eram disponibilizados para os alunos em meios tradicionais através de livros 
VI Congresso Brasileiro de Informática na Educação (CBIE 2017)

Anais do XXVIII Simpósio Brasileiro de Informática na Educação (SBIE 2017)

didáticos ou anotações no caderno, o que tornava a aprendizagem mecânica e desmotivadora. Além disso, esses métodos tradicionais de ensino nem sempre mantém o aluno focado na aula, tornando-o disperso e reduzindo a aprendizagem na sala de aula.

Com o surgimento dos conceitos construtivistas e das Novas Tecnologias de Informação e Comunicação (NTICs), uma nova dinâmica de relação entre professor e aluno se estabeleceu e foi mais além ao exigir que o aluno assumisse seu papel na construção do conhecimento [Farias et al, 2015]. Além disso, as novas tecnologias digitais têm tornado a educação construtivista e informatizada oferecendo um campo fértil de exploração na busca pela consolidação de ambientes adaptados às características diferenciadas de aprendizagem dos alunos enriquecendo assim a criação de simulações que reproduzem circunstâncias reais. A integração da tecnologia no processo ensino-aprendizagem vem sendo uma questão bastante discutida na área educacional, pois, tem provocado mudanças significativas nos modelos educativos [Kensi, 2007].

Diante disso, alguns estudantes estão buscando formas alternativas de aprendizagem com o uso de metodologias ativas e interativas como, por exemplo, jogos digitais. Jogos são elaborados com intuito de ensinar as pessoas, um determinado assunto, conceito, desenvolver uma habilidade física e/ou lógica, entre outros [Lopes et al, 2015]. Na educação, o potencial da gamificação é imenso, ela funciona para despertar interesse dos alunos, aumentar a participação dos alunos na sala de aula, desenvolver criatividade e autonomia, promover diálogo e resolver situações-problema, principalmente utilizando recursos como o uso de novas tecnologias [Lorenzoni, 2016].

Nos cursos de Saúde, o processo de ensino-aprendizagem é um pouco diferente daquele realizado em salas de aula, pois quanto aos conteúdos, integram-se os de caráter técnico-informativos às questões formativas éticas, morais, psicológicas, ligadas às relações sociais estabelecidas [Garcia, 2001]. Além disso, o ensino de saúde também sofre dos mesmos problemas da falta de engajamento, interesse e interatividade, pois o conteúdo das disciplinas de saúde é muitos extensos, complexos e cansativos. Um exemplo é o conteúdo da disciplina de Anatomia Humana que é uma das matérias que mais exige do aluno, uma vez que o aluno precisa entender o funcionamento $\mathrm{e}$ memorizar todas as partes dos sistemas do corpo humano, o sistema urinário, por exemplo, possui muitas funções, elementos e características, que lembrar o nome de todos não é tarefa fácil.

Além disso, transformar conteúdos estáticos numa realidade mais próxima do cotidiano do aluno é um desafio que está presente nos dias atuais. A realidade Virtual e Aumentada é uma tecnologia que surgiu para tornar o conteúdo o mais próximo da realidade através da imersão do aluno num mundo virtual onde ele não conseguiria alcançar. Ela é perfeita para o desenvolvimento de aplicações interativas onde o usuário deve possuir alto nível de imersão [Araújo et al, 1999]. Com o uso de tal tecnologia, o aluno pode imergir dentro do corpo humano, visualizar e interagir com todas suas partes em 3D [Colpani et al, 2016].

Dessa maneira, este artigo tem como objetivo apresentar a metodologia de Design centrado no usuário e avaliação de usabilidade de um ambiente virtual de apoio 
VI Congresso Brasileiro de Informática na Educação (CBIE 2017)

Anais do XXVIII Simpósio Brasileiro de Informática na Educação (SBIE 2017)

ao processo ensino-aprendizagem de anatomia humana baseado nos conceitos de gamificação e realidade virtual para alunos de saúde.

\section{Metodologia}

O método utilizado na pesquisa foi baseado nas práticas de engenharia de software e Design centrado no usuário (Human-Centered Design) que é um processo de design e avaliação composto por um conjunto de técnicas utilizadas para criar novas soluções e modos de interação [HCD, 2017]. As técnicas utilizadas na pesquisa foram desde análise bibliográfica, levantamento dos competidores, entrevista com usuários para definir os requisitos necessários para criação do game, análise de contexto, personas e cenários com especialistas para geração de protótipos interativos utilizando a técnica de prototipagem de baixa e alta fidelidade assim como diagramação UML e casos de uso na implementação do game.

O estudo também foi baseado em uma abordagem qualitativa onde teve caráter exploratório. A variável qualitativa usada para capturar a usabilidade do jogo e a percepção de usuários no estudo se baseou na avaliação de usabilidade e interpretação sobre o objeto estudado através da técnica de inspeção de usabilidade [Nielsen, 2004] e thinkaloud [Jaspers, 2004]. Os instrumentos utilizados na coleta dos dados foram questionários, entrevistas e gravações.

\section{1- Participantes}

A população foi composta por alunos de graduação dos cursos de Saúde de uma Faculdade da Região metropolitana de Recife que concordaram em participar do experimento. Nós conseguimos apenas 07 (sete) alunos para realizar o teste de usabilidade, mas segundo Nielsen (2012), um usuário pode identificar aproximadamente $30 \%$ dos problemas de usabilidade de uma interface. Nielsen apresentou em seus estudos que, a partir de cinco (5) usuários, o crescente número de falhas identificadas não representa um ganho significativo. Além disso, a porcentagem de erros de usabilidade encontrados por um grupo de cinco usuários é de $85 \%$, o que representa a melhor relação custo-benefício [Nielsen, 2012]. Para minimizar o viés, a informação contida nos termos de participação explicou que a participação seria voluntária e que as informações relatadas eram confidenciais, e somente armazenado pelos pesquisadores.

\section{2- Contexto}

Este projeto fez parte da disciplina de Interação Humano-Computador, do curso de Jogos Digitais de uma faculdade da cidade de recife, Pernambuco. Para o desenvolvimento do projeto da disciplina, o professor sugeriu a interdisciplinaridade entre as áreas de Tecnologia da Informação e Saúde. O projeto da disciplina era o desenvolvimento e análise das interfaces de um jogo centrado no usuário da área de saúde. O jogo foi desenvolvido numa plataforma de desenvolvimento de jogos Unity juntamente com o google cardboard para torna uma solução de game de baixo custo e acessível a todos os estudantes. 
VI Congresso Brasileiro de Informática na Educação (CBIE 2017)

Anais do XXVIII Simpósio Brasileiro de Informática na Educação (SBIE 2017)

\subsection{Processo de design de Interface centrado no Usuário}

O processo design de interface centrado no usuário da plataforma foi uma das etapas da metodologia de desenvolvimento de software e foi subdividida em 06 etapas:

- Escolha do Problema: O Professor passou alguns conteúdos da área de saúde que apresentavam algum tipo de dificuldade no processo de aprendizagem com os alunos. Diante dos conteúdos expostos escolhemos o assunto sobre o funcionamento e anatomia do sistema urinário.

- Análise de usuário: Depois de escolhido o conteúdo, fizermos um questionário de pesquisa de analisar e entender o perfil tecnológico e cognitivo do nosso usuário que é o aluno de graduação da área de saúde.

- Geração de Solução: Com base na análise dos resultados do perfil do usuário, utilizamos a técnica de Brainstorm juntamente com algumas reuniões para elencar as ideias de cada um da equipe e discutir possíveis soluções de jogo que atendesse a solucionar as dificuldades de aprendizado.

- Seleção de Ideias: Selecionamos as melhores ideias produzidas na etapa anterior nas quais são avaliadas e selecionadas, com base em sua viabilidade, utilidade, custo, etc. E após colocamos as melhores numa só solução.

- Prototipação: a solução foi desenvolvida inicialmente com protótipos de baixa fidelidade, mas devido ao tipo de tecnologia escolhida (Realidade Virtual) foi necessário gerar um protótipo navegável de alta fidelidade da solução para que possa ser testado.

- Validação: o protótipo é testado para avaliar sua eficiência em resolver o problema e são coletadas novas informações para melhorar a solução e resolver os problemas encontrados.

\subsection{Avaliação de Usabilidade}

A avaliação de usabilidade foi realizada inicialmente com os ensaios de usabilidade, onde se testou as funcionalidades da plataforma Anatomia Digital para identificar possíveis problemas de interação antes que o projeto seja implementado completamente e terminado. Essa avaliação é feita a partir das técnicas objetivas e prospectivas baseadas na observação e monitoramento das interações dos usuários e por coleta de opiniões dos usuários.

\section{Resultados e discussão}

O objetivo do projeto foi o desenvolvimento e análise da interface e usabilidade de um jogo educacional que ajudasse os alunos a entenderem o funcionamento da anatomia do sistema urinário, no qual os alunos deveriam aprender quais substâncias formam a urina e quais substâncias voltam ao corpo através da corrente sanguínea. O Jogo educacional é um ambiente virtual desenvolvido baseado no processo de design centrado no usuário onde os alunos com a supervisão dos professores, aprendem através de minigames os sistemas do corpo humano. Para uma melhor interação e imersão, decidimos a utilização da tecnologia VR com o controle de movimentação para facilitar o aprendizado dos alunos. 
VI Congresso Brasileiro de Informática na Educação (CBIE 2017)

Anais do XXVIII Simpósio Brasileiro de Informática na Educação (SBIE 2017)

\subsection{Perfil dos Usuários}

O questionário da análise do perfil tecnológico e cognitivo do usuário foi realizado a partir de uma pesquisa online pelo Google Formulário com a finalidade de identificar as características tecnológicas e cognitivas de aprendizagem dos alunos de saúde e quais dificuldades e necessidades que eles têm em relação para aprender o assunto. De acordo com a análise dos resultados do questionário do perfil do usuário, definiu-se que o usuário tinha as seguintes características:

- Faixa Etária: 79,2\% dos usuários estão entre 19 e 30 anos.

- Gênero: 83,2\% dos usuários é do sexo Feminino.

- Renda Familiar: 50,4\% dos usuários tem renda familiar de 2 à 3 salários mínimos.

- Curso: 61,6\% dos usuários se encontram fazendo o curso de Enfermagem.

- Dispositivos: $89,6 \%$ dos usuários disseram que usam o computador e/ou smartphone no dia a dia sendo que 69,6\% deles usam mais o smartphone.

- Principais dificuldades sobre o aprendizado do assunto sobre o sistema urinário:

- Entendimento do Assunto Sistema Urinário:

○ 7,7\% dos usuários não sabiam nada sobre o assunto;

- $15,4 \%$ dos usuários sabiam um pouco superficialmente o assunto;

- $38,5 \%$ dos usuários sabiam mais ou menos sobre o assunto;

- $23,1 \%$ dos usuários sabiam razoavelmente o assunto; e

○ $5,4 \%$ dos usuários sabiam tudo sobre o assunto.

- Alternativa para o aprendizado em geral: $72 \%$ dos usuários responderam que prefere um ambiente Virtual como alternativa para o aprendizado.

- Facilidade em aprender: $84 \%$ dos usuários disseram que tem facilidade em aprender com um vídeo, com textos ou animações.

\subsection{Análise de Concorrentes}

Durante a fase de análise de concorrentes foram encontrados 3 (três) concorrentes diretos para o projeto (Tabela 1): 3D Organon VR Anatomy (Software sobre anatomia dos sistemas em 3D para smartphones), Anatomyou (Software 3D sobre anatomia do corpo humano para smartphones) e Virtual Anatomy Simulation (Software 3D sobre a estrutura e os órgãos do corpo humano usando tecnologia AR e VR).

Tabela 1. Análise de concorrentes

\begin{tabular}{|c|c|c|c|}
\hline Concorrente & $\begin{array}{c}\text { 3D Organon VR } \\
\text { Anatomy }\end{array}$ & Anatomyou & $\begin{array}{c}\text { Virtual Anatomy } \\
\text { Simulation }\end{array}$ \\
\hline Descrição & $\begin{array}{c}\text { Aplicativo sobre } \\
\text { o primeiro atlas } \\
\text { de anatomia } \\
\text { sobre sistemas } \\
\text { do corpo } \\
\text { humano em VR }\end{array}$ & $\begin{array}{c}\text { Aplicativo 3D } \\
\text { imersivo que irá } \\
\text { ajudar a entender a } \\
\text { anatomia do corpo } \\
\text { humano com visão } \\
\text { em } 360^{\circ} .\end{array}$ & $\begin{array}{c}\text { O Virtual Anatomy } \\
\text { pela EONor, desenvolvido } \\
\text { software de RV e RA } \\
\text { para os alunos } \\
\text { aprenderem as }\end{array}$ \\
\hline
\end{tabular}


VI Congresso Brasileiro de Informática na Educação (CBIE 2017)

Anais do XXVIII Simpósio Brasileiro de Informática na Educação (SBIE 2017)

\begin{tabular}{|c|c|c|c|}
\hline & $\begin{array}{l}\text { completo do } \\
\text { mundo. }\end{array}$ & & $\begin{array}{l}\text { estruturas e os órgãos } \\
\text { do corpo humano. }\end{array}$ \\
\hline Vantagens & $\begin{array}{c}\text { Oferece um } \\
\text { catálogo } \\
\text { completo de } \\
\text { sistemas e suas } \\
\text { anatomias com } \\
\text { suporte a } \\
\text { tecnologia VR. }\end{array}$ & $\begin{array}{l}\text { Anatomyou é baseado } \\
\text { em tecnologia 3D- } \\
\text { imersiva para } \\
\text { fornecer uma maneira } \\
\text { fácil de aprender } \\
\text { anatomia focada em } \\
\text { técnicas } \\
\text { minimamente } \\
\text { invasivas. }\end{array}$ & $\begin{array}{l}\text { O Virtual Anatomy } \\
\text { Simulator traz a } \\
\text { tecnologia VR e AR } \\
\text { para que os alunos } \\
\text { aprendam de forma } \\
\text { imersiva a estrutura do } \\
\text { corpo humano. }\end{array}$ \\
\hline Desvantagens & \multicolumn{3}{|c|}{$\begin{array}{l}\text { Os aplicativos oferecem um conteúdo e recurso limitado a alunos } \\
\text { profissionais da área, com temática realista em todos os detalhes. }\end{array}$} \\
\hline
\end{tabular}

De acordo com a tabela 1 , podemos observar que, quando relacionamos esses três produtos com a plataforma de Anatomia Digital, a plataforma possui as mesmas características tecnológicas de todos os concorrentes, mas com a diferença que a plataforma é baseada nas metodologias de gamificação com minigames interativos e também com metodologias pedagógicas ativas onde os alunos têm liberdade de navegar e escolher o que vai fazer dentro do jogo que vai desde a busca de informações para seu aprendizado. Além disso, os professores podem visualizar os rendimentos dos seus alunos após utilizarem os jogos, através de relatórios.

\subsection{Escolha da Solução}

Para encontrar uma solução para melhorar o interesse, motivação e a taxa cognitiva de aprendizado dos alunos em relação ao conteúdo sobre o funcionamento e anatomia do sistema urinário, dificuldade sugerida pela professora de Saúde, foi utilizado a técnica de Brainstorm para desenvolver ideias e logo após houve a seleção de ideias, para selecionar a(as) ideia(as) para desenvolver uma solução para a dificuldade de aprendizagem.

A técnica de Brainstorm foi utilizada com auxílio de um professor de computação gráfica e modelação $3 \mathrm{D}$, que utilizou a técnica com o grupo para desenvolver ideias para o problema. No final do Brainstorm se destacaram três ideias diferentes para desenvolver a solução. Na etapa de seleção de ideia(as) foi utilizado a técnica de votação para escolher a melhor ideia e depois de escolher a melhor ideia foi desenvolvido um sistema de acabamento para finalizar todos os pontos principais da ideia escolhida. Pela votação do grupo foi escolhida a Ideia 02, por votação unânime que vai ser descrita na sessão 3.1 .

\subsection{Prototipação da Ideia}

Com a ideia selecionada, houve a realização de protótipos para entender todas funcionalidades da plataforma e identificar os primeiros erros de usabilidade e das funcionalidades. Os protótipos foram criados a partir do uso do software free da 
VI Congresso Brasileiro de Informática na Educação (CBIE 2017)

Anais do XXVIII Simpósio Brasileiro de Informática na Educação (SBIE 2017)

microsoft, o Paint, onde se desenvolveu protótipos do ambiente virtual e do minigame relacionado ao sistema urinário (Figura 1).

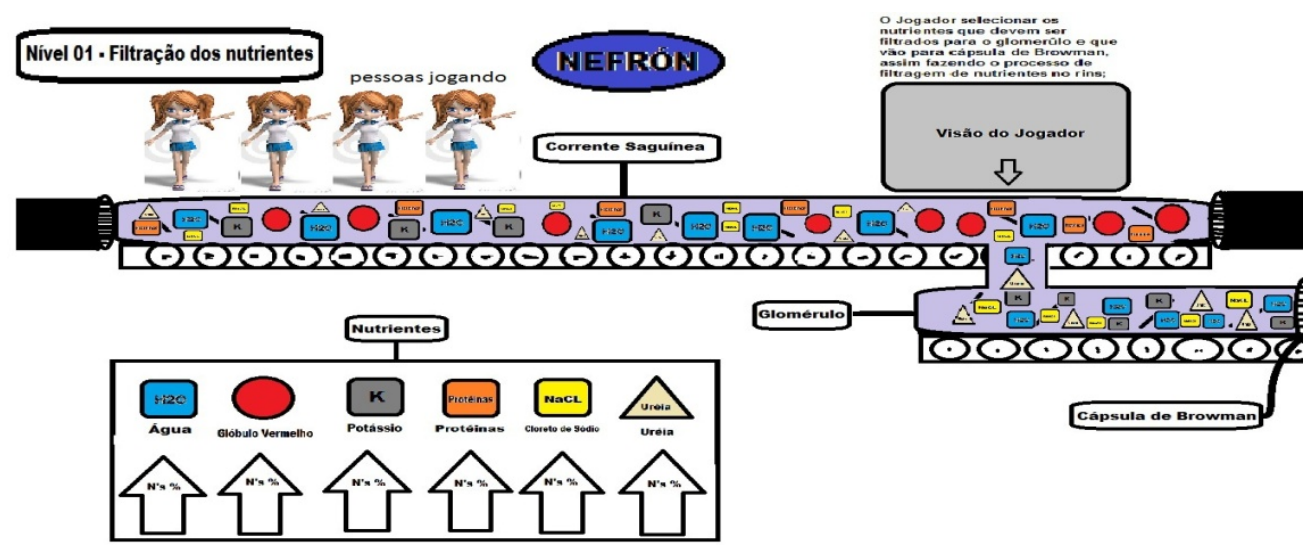

Figura 1: Protótipo da primeira tela de gameplay do minigame.

\subsection{Análise da tarefa}

Durante a criação dos protótipos, é realizado o planejamento das tarefas que o aluno irá realizar durante o teste de usabilidade. Na primeira versão do protótipo foram definidas duas tarefas para o aluno realizar, são elas:

- Tarefa 01 - Ambiente Virtual: o aluno tem que fazer o cadastro e login no ambiente virtual para que depois eles aceitassem o convite do professor para entrar numa sala de aula virtual onde iriam iniciar o minigame.

- Tarefa 02 - Minigame: os usuários têm que abrir o aplicativo Anatomia Digital no smartphone e fazer o processo de login com os dados de usuário do ambiente no aplicativo. Depois eles iriam conectar o smartphone que contém a aplicação, com os óculos de realidade virtual e com controle de movimento. Os alunos teriam que assistir o tutorial do minigame para entender como ele funciona e depois teriam que jogar o gameplay até que eles conseguissem terminar ou não o minigame.

\subsection{Descrição do jogo}

A plataforma Anatomia Digital foi dividida em dois ambientes distintos: o gerenciador acadêmico web e o aplicativo de Realidade Virtual. O gerenciador acadêmico web é um sistema onde os alunos e professores da área de saúde vão se cadastrar online. Nelo, o professor vai ter a disponibilidade de criar salas temáticas online para hospedar seus alunos e escolher qual minigame irá utilizar. Após o cadastro, os alunos se conectam nas salas a partir de convites feitos pelo administrador da sala, no caso o professor. Cada minigames representa um sistema do corpo humano e vão estar disponível para download nas lojas de aplicativos dos smartphones que tenha suporte à tecnologia VR. Após abrir o aplicativo no smartphone, o aluno terá que se logar no aplicativo com os mesmos dados que se cadastraram no ambiente. A seguir, os alunos vão começar a jogar o minigame inserindo o smartphone nos óculos de realidade virtual. 
VI Congresso Brasileiro de Informática na Educação (CBIE 2017)

Anais do XXVIII Simpósio Brasileiro de Informática na Educação (SBIE 2017)

Para jogar o minigame, os alunos utilizaram um controle de movimento para interagir com o minigame. Ao iniciar o jogo os usuários serão recepcionados pela assistente virtual Amora na qual vai solicitar que o usuário assista ao tutorial do jogo (Figura 2a). Depois de assistir o tutorial do jogo, o usuário irá selecionar a sala temática que irá jogar o minigame através de uma tela de seleção, onde vai ficar um corpo humano com cada sistema que o compõem (Figura $2 b$ ).

Quando o minigame for iniciado, vai haver instruções sobre o funcionamento do minigame e do sistema escolhido. No minigame do sistema urinário (Figura 2c), os alunos verão duas esteiras, uma da corrente sanguínea e outra da bexiga por onde as substâncias do sistema urinário irão passar. Quando os alunos miram com controle nas substâncias aparecerá uma descrição com o nome da substância. Em cima da esteira da corrente sanguínea vão surgindo as substâncias que percorreram a esteira até chegar no coletor da corrente sanguínea. Nesse momento, os alunos terão que distinguir quais são as substâncias boas e ruins e selecionar as substâncias ruins para colocarem em outra esteira que representa o túbulo coletor que encaminhará as substâncias coletadas para a bexiga, assim formando a urina. As outras substâncias boas que não foram selecionadas irão sendo encaminhadas para o túbulo coletor da corrente sanguíneo.

$\mathrm{Na}$ parte superior da tela será informado o tempo que os alunos têm para realizar as atividades, a sua pontuação, uma tabela detalhando quais substâncias são ruins e boas e outra informando o excesso por porcentagem das substâncias boas, para que os alunos recolham as substâncias boas que estão em excesso no corpo (Figura $2 \mathrm{~d}$ ).

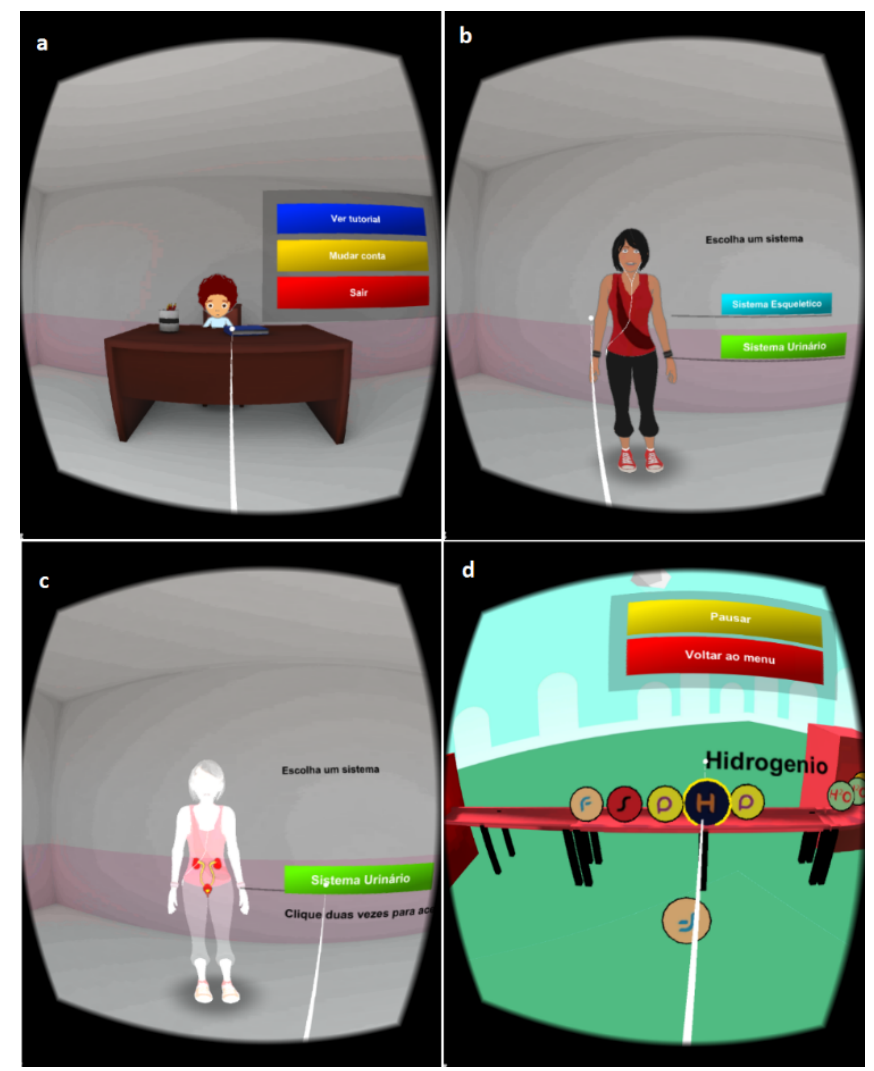

Figura 2 - Controle de movimento para interagir com o minigame 
O jogo termina quando os alunos conseguirem encher a bexiga, formando a urina com as substâncias recolhidas ou quando o aluno não consegue separar as substâncias más e elas vão para corrente sanguínea completando $100 \%$.

\subsection{Problemas de Usabilidade}

Para captação de dados para dar o resultado do teste de usabilidade, foi feito um ensaio de usabilidade em que os usuários utilizavam os serviços da plataforma: o ambiente virtual e o aplicativo, em que foram capturados imagem das telas dos dispositivos e captação dos comportamentos e reações dos usuários através de filmagem. Os dados coletados dos ensaios levaram aos seguintes resultados:

Em relação ao teste de usabilidade da atividade 1: todos os usuários não tiveram quaisquer problemas ao se conectar com o ambiente, executando as tarefas em tempo médio de 2 minutos e 30 segundos;

Já em relação ao teste de usabilidade da atividade 2: os usuários detectaram alguns problemas no aplicativo, no minigame e na utilização dos óculos de realidade virtual, como:

- Dificuldades no manuseio do controle de movimento enquanto utilizavam os óculos de realidade virtual durante a execução do aplicativo Anatomia Digital.

- Perda de noção espacial e de posicionamento no gameplay do minigame. Solução:

- Ausência de um tutorial interativo detalhado com mais imagens, animação ou um vídeo descrevendo o funcionamento do minigame quando acessa o sistema urinário.

- Ausência de feedback durante o gameplay em relação a coleta das substâncias más da corrente sanguínea para a bexiga, de quando pegava a substância da corrente e colocava no caminho para bexiga, o jogo não dava resposta de que a substância foi coletada, apenas que a substância aparecia ao ser coletada pelo usuário abaixo dele.

Além desses problemas de usabilidade, alguns usuários relataram um minigame era muito simples, pois abordava apenas a parte fisiológica do sistema urinário e acabava deixando de lado o aspecto estrutural do sistema. Em relação ao tempo de uso do aplicativo e do gameplay do minigame, os usuários de início sentiram dificuldade de evoluir nas tarefas por estarem utilizando o aplicativo pelo VR, mas logo em seguida, quando aprenderam o tutorial do aplicativo para utilizar o controle, os usuários demonstraram conformidade e conseguiram cumprir as tarefas, em um tempo médio de 12 minutos.

No geral o teste de usabilidade na plataforma Anatomia Digital ocorreu de forma normal e agradável na visão dos usuários, que demonstraram positividade para o futuro do projeto. 


\section{Conclusão}

O projeto da plataforma Anatomia Digital permitiu desenvolver uma solução para a dificuldade de aprendizado dos alunos de saúde utilizando novas tecnologias em formato gamificado, imersivo e interativo. A plataforma fez com que os estudantes experienciassem novas tecnologias como a Realidade Virtual, aumentando a imersão nos conteúdos do ensino de saúde, trazendo-os para dentro dos sistemas e seus subsistemas do corpo humano e fazendo com que eles se sintam parte do sistema. Além disso, a metodologia utilizada proporcionou um processo de aprendizado mais imersivo, claro e principalmente mais interativo para os estudantes, ocasionando em um estímulo a mais para eles na hora dos estudos.

Com os resultados, observamos que o uso da tecnologia de Realidade Virtual integrado no processo de ensino-aprendizagem dos alunos de saúde não era um hábito comum, mas essa integração forneceu benefícios significativos para aprender, para melhorar o processo de ensino, facilitando a visualização das informações de forma mais realística e imersivas. O objetivo do projeto não é apenas ofertar novas formas de aprendizado, mas também construir uma metodologia pedagógica forma de imersão e interação num ambiente mais realístico do conteúdo que estava sendo aprendido.

Apesar de apenas uma pequena quantidade dos usuários participou do experimento, os resultados deste estudo corroboram não só na análise de usabilidade e na experiência do usuário na utilização da plataforma como suporte ao processo de ensino-aprendizagem como também na validação da eficiência da integração de metodologias de aprendizagem com tecnologias de Realidade Virtual para o aprendizado de alunos de cursos de saúde.

Além disso, houve evidências de que a preferência de todos os usuários em favor do uso da plataforma como ferramenta de apoio ensino e relacioná-la com a sua simplicidade e a possibilidade de imergir dentro de um ambiente virtual do corpo humano e visualizar em 3D seus elementos como evidenciado pelas palavras de alguns participantes na seção de análise de satisfação. Em relação variável usabilidade, os alunos, apesar de não ter o perfil tecnológico, acharam a plataforma fácil de usar como ferramenta de suporte para a aprendizagem e se sentiam mais satisfeitos e atualizado com o conteúdo da disciplina.

\section{Referências}

Araujo, R. B., Battaiola, A. L., Goyos, C. (1999). Exploração do Uso de Realidade Virtual no Aprendizado de Habilidades Acadêmicas para o Ensino Fundamental. Revista Brasileira de Informática na Educação, $\mathrm{N}^{\circ}$ 04, Disponível em: <http://www.br-ie.org/pub/index.php/rbie/article/view/2291/2053>.

Colpani, R., Homen, M. R. P. (2016). Realidade Aumentada e Gamificação na Educação: Uma aplicação para auxiliar no processo de aprendizagem de alunos com deficiência intelectual. Revista Brasileira de Informática na Educação, Vol 24, No 01, Disponível em: <http://www.br-ie.org/pub/index.php/rbie/article/view/3347/4489>.

Farias, P. A. M., Martin, A. L. A. R., Cristo, C. S. (2015). Aprendizagem Ativa na Educação em Saúde: Percurso Histórico e Aplicações. Revista Brasileira de 
VI Congresso Brasileiro de Informática na Educação (CBIE 2017)

Anais do XXVIII Simpósio Brasileiro de Informática na Educação (SBIE 2017)

Educação Médica, 39(1), 143-150.

Garcia, M. A. A. (2001). Saber, agir e educar: o ensino-aprendizagem em serviços de Saúde. Interface - Comunicação, Saúde, Educação, 5(8), 89-100. https://dx.doi.org/10.1590/S1414-32832001000100007

HCD Methods. Disponível em: <http://www.hcdconnect.org/methods>. Acesso em: 17 jul. 2017

Jaspers M. W., Steen T., van den Bos C, Geenen M. (2004). The think aloud method: a guide to user interface design. Int J Med Inform. Nov;73(11-12):781-95.

Kensi, V. M. (2007). Educação e Tecnologias: O novo ritmo da informação. Campinas, SP: Papirus, 2007, $6^{\mathrm{a}}$ ed.

Lopes, R. A.; Toda, A. M.; Brancher, D. B. (2015) Um estudo preliminar sobre elementos extrínsecos e intrínsecos do processo de Gamification. Revista Brasileira de Informática na Educação, Volume 23, No 03.

Lorenzoni, M. (2016). Gamificação: Como é e como pode transformar a aprendizagem. InfoGeekie, Disponível em: <http://info.geekie.com.br/gamificacao/>.

Nielsen, J. (1993). Usability Heuristics. In: NIELSEN, J. Usability Engineering. San Francisco: Morgan Kaufmann Publishers Inc. p. 155-200. 